\title{
Pregnancy rates after vitrification, warming and transfer of equine embryos
}

\author{
G.H.M. Araujo ${ }^{a}, *$, A.N. Rocha Filho ${ }^{b}$, S.D. Burns ${ }^{b}$, C.M. Burns ${ }^{b}$, C.F. Moya-Araujo ${ }^{c}$, C. Meira ${ }^{a}$ \\ a School of Veterinary Medicine and Animal Science, Sao Paulo State University, UNESP, Dist. Rubiao Jr. s/n, 18618-970, Botucatu, SP, Brazil \\ b Burns Ranch, Menifee, CA, USA \\ c School of Veterinary Medicine, FIO, Ourinhos, SP, Brazil
}

\section{Introduction}

The use of low temperature techniques to preserve equine embryos has become essential to the equine industry, and cooling, freezing and vitrification are successful techniques for assisted reproduction in this species. Vitrification consists of a short incubation time in high concentrations of cryoprotectants followed by direct plunging into liquid nitrogen to prevent ice crystal formation. The technique aims to produce embryos for future use, for example when a better recipient is available, or to optimize the production of foals when the specific breed associations establish limits on the breeding season months.

\section{Materials and methods}

Data were collected during the 2008 and 2009 breeding seasons in the northern hemisphere using Quarter Horse mares, ranging from 4 to 18 years of age. Mare's reproductive tracts were examined by ultrasonography daily for detection of ovulation prior to breeding. On day 6 post-ovulation, embryos were recovered by nonsurgical uterine flush using Dulbecco's phosphate buffered saline (D-PBS, D4031, SIGMA-ALDRICH, St. Louis, MO, USA) with $1 \%$ fetal bovine serum (FBS, HYCLONE, Logan, UT, USA). Upon recovery, only morulae and early blastocysts with excellent or good morphology were used $(n=64)$. Embryos were washed in D-PBS with 10\% FBS before exposure to

\footnotetext{
is This paper is part of the supplement entitled "Proceedings of the Tenth International Symposium on Equine Reproduction”, Guest Edited by Margaret J. Evans.

* Corresponding author. Tel.: +55 1438116249 .

E-mail address: ghmaraujo@uol.com.br (G.H.M. Araujo).
}

vitrification solutions (Syngro ${ }^{\mathrm{TM}}$ Vitrification Kit; Bioniche Animal Health USA, Pullman, WA, USA) at room temperature. Embryos were placed in a four-well dish containing VS1 (5 min), moved to VS2 ( $5 \mathrm{~min}$ ) and then transferred into VS3. The embryo in VS3 was loaded in the center of a $0.25-\mathrm{mL}$ straw (IMV, Maple Grove, MN, USA), separated by two air bubbles from two columns of diluent. The embryo was in contact with VS3 for approximately $45 \mathrm{~s}$ before the straw was sealed and placed in a cooled plastic goblet surrounded by liquid nitrogen for $1 \mathrm{~min}$ before being submerged. Straws were stored in liquid nitrogen until warming and transfer. To warm embryos, the straw was held in air for $10 \mathrm{sec}$ and then immersed in a $22^{\circ} \mathrm{C}$ water bath for $10 \mathrm{~s}$. The straw was flicked vigorously 5-7 times, opened and its content transferred to a small dish containing holding solution (Emcare ${ }^{\mathrm{TM}}$; Auckland, New Zealand) in which embryos were washed and held for at least $5 \mathrm{~min}$. This modification in the dilution procedure was done to allow evaluation and stabilization of the embryo. Embryos were packaged in $0.25 \mathrm{~mL}$ straws and loaded in a "French gun" before nonsurgical transfer into the uteri of recipients that had ovulated 5 or 6 days before. Fisher's exact test and Chi-square test were used to compare pregnancy and embryonic loss data.

\section{Results and discussion}

Pregnancy rates at 15 days were 67\% (16/24) in 2008 and $55 \%(22 / 40)$ in 2009 (59\% overall; 38/64) while embryonic loss rates until 45 days of gestation were $12.5 \%(2 / 16)$ in 2008 and $9.1 \%(2 / 22)$ in 2009. There was no effect of season $(P>0.05)$. When compared to vitrified embyros, transfer of fresh embryos yielded higher $(P<0.05)$ pregnancy rates (2008: 87.6\% (92/105); 2009: 78.2\% (104/133) but similar 
$(P>0.05)$ pregnancy loss rates (2008: 9.8\% (9/92); 2009: $10.5 \%(11 / 104))$. In the present study, vitrified morulae and early blastocysts produced similar pregnancy rates as described in the literature, demonstrating that the procedure can be used successfully under field conditions in commercial operations. Although direct comparisons with the regular vitrification methodology were not made, exposing previously vitrified embryos to holding solution prior to transfer did not seem to be detrimental.

\section{Conflict of interest}

None.

\section{Acknowledgment}

This work is supported by Burns Ranch Inc. - CA/USA; FAPESP - SP/Brazil. 